\title{
Optimization of sentinel lymph node biopsy in breast cancer using an operative gamma camera Carole Mathelin*1, Samuel Salvador ${ }^{2}$, Sabrina Croce ${ }^{3}$, Norosoa Andriamisandratsoa ${ }^{4}$, Daniel Huss ${ }^{2}$ and Jean-Louis Guyonnet ${ }^{2}$
}

\author{
Address: ${ }^{1}$ Service de Gynécologie-Obstétrique, Hôpital Civil, 1 place de l'Hôpital. F-67091 Strasbourg Cedex, France, ${ }^{2}$ Institut Pluridisciplinaire \\ Hubert Curien. UMR 7178 - CNRS/IN2P3 et ULP. BP 28 - 23 rue du Loess. F-67037 Strasbourg Cedex 02, France, ${ }^{3}$ Département d'anatomie- \\ pathologique. Hôpital de Hautepierre, Avenue Molière. F-67200 Strasbourg Cedex, France and ${ }^{4}$ Service de Médecine Nucléaire. Hôpital Civil, 1 \\ place de l'Hôpital. F-67091 Strasbourg Cedex, France \\ Email: Carole Mathelin* - carole.mathelin@chru-strasbourg.fr; Samuel Salvador - dark_bass@hotmail.com; \\ Sabrina Croce - sabrina.croce@chru-strasbourg.fr; Norosoa Andriamisandratsoa - norosoa.andrianatoandro@chru-strasbourg.fr; \\ Daniel Huss - daniel.huss@IReS.in2p3.fr; Jean-Louis Guyonnet - jean-louis.guyonnet@IReS.in2p3.fr \\ * Corresponding author
}

Published: 17 November 2007

World Journal of Surgical Oncology 2007, 5:132 doi:10.1186/1477-7819-5-132

This article is available from: http://www.wjso.com/content/5/I// 32

(C) 2007 Mathelin et al; licensee BioMed Central Ltd.

This is an Open Access article distributed under the terms of the Creative Commons Attribution License (http://creativecommons.org/licenses/by/2.0), which permits unrestricted use, distribution, and reproduction in any medium, provided the original work is properly cited.

\begin{abstract}
Background: Sentinel lymph node (SLN) procedure is now a widely accepted method of LN staging in selected invasive breast cancers (unifocal, size $\leq 2 \mathrm{~cm}$, clinically N0, without previous treatment). Complete axillary clearance is no longer needed if the SLN is negative. However, the oncological safety of this procedure remains to be addressed in randomized clinical trials. One main pitfall is the failure to visualize SLN, resulting in incorrect tumor staging, leading to suboptimal treatment or axillary recurrence. Operative gamma cameras have therefore been developed to optimize the SLN visualization and the quality control of surgery.
\end{abstract}

Case presentation: A 44-year-old female patient with a $14-\mathrm{mm}$ infiltrative ductal carcinoma underwent the SLN procedure. An operative gamma camera was used during and after the surgery. The conventional lymphoscintigraphy showed only one SLN, which was also detected by the operative gamma camera, then removed and measured $(9.6 \mathrm{kBq})$. It was analyzed by frozen sections, showing no cancer cells. During this analysis, the exploration of the axillary area with the operative gamma camera enabled the identification of a second $S L N$ with low activity $(0.5 \mathrm{kBq})$ that conventional lymphoscintigraphy, surgical probe and blue staining had failed to visualize. Histological examination revealed a macrometastasis. Axillary clearance was then performed, followed by a postoperative image proving that no SLN remained. Therefore, the use of the operative gamma camera prevented an under-estimation of staging which would have resulted in a suboptimal treatment for this patient.

Conclusion: This case report illustrates that an efficient operative gamma camera may be able to decrease the risk of false negative rate of the SLN procedure, and could be an additional tool to control the quality of the surgery.

Trial Registration: ClinicalTrials.gov Identifier: NCT00357487 


\section{Background}

As lymph node (LN) metastasis is one of the most important prognostic factors for survival, the assessment of regional LN is essential in the staging of breast cancer, ascertaining a prognosis, and determining of optimal adjuvant treatments. The sentinel node (SLN) procedure consists of recognizing and removing the first LN that filter lymphatic fluid from the tumor. It is now a widely accepted method of LN staging in selected invasive breast cancer (unifocal, size $\leq 2 \mathrm{~cm}$, clinically No, without previous treatment). The complete axillary clearance is no longer needed if the SLN is normal [1]. However, its oncological safety remains to be addressed in randomized clinical trials [2]. One main pitfall is the failure to visualize SLN, resulting in incorrect tumor staging, leading to suboptimal treatment or axillary recurrence. Several factors including advanced age, high body mass index, massive metastasis invasion [3] or tumor location other than upper outer quadrant [4] may contribute to SLN non-visualization.

Operative gamma cameras (OGC) have therefore been developed to optimize the SLN visualization and the quality control of surgery [5-10]. In our ongoing clinical trial [11], an OGC, named CarolIReS, with a current field of view (FOV) of $50 \times 50 \mathrm{~mm}^{2}$ is being used [12]. It is made of a lead parallel collimator, a thin $\mathrm{Gd}_{2} \mathrm{SiO}_{5}$ :Ce scintillating crystal and a multianode photomultiplier $(10 \mathrm{~mm}$ spatial resolution and $2.2 \mathrm{cps}^{\mathrm{kBq}}{ }^{-1}$ sensitivity for the

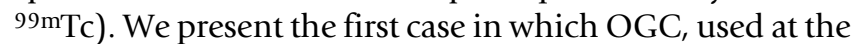
end of the surgery, enabled the detection of a residual metastatic SLN.

\section{Case presentation}

A 44-year-old female patient with a $14 \mathrm{~mm}$ infiltrative ductal carcinoma, Scarf-Bloom-Richardson grade 2 (diagnosed by core biopsy) was enrolled in the clinical study [11] after informed consent had been obtained. She was free of chemotherapy, loco-regional radiotherapy, prevalent axillary LN and her body mass index was normal (21 $\mathrm{kg} / \mathrm{m}^{2}$ ). The tumor was located in the upper outer quadrant of the left breast. The SLN procedure was initiated the day prior to the surgery using 4 subareolar injections of rhenium sulfur colloidal and technetium $\left({ }^{99 \mathrm{mTc}}\right)(0.4 \mathrm{ml}$, $22 \mathrm{MBq}(595 \mu \mathrm{Ci})$, Cis bio international ${ }^{\oplus}$, France). Scintigraphic images (anterior, lateral and oblique views), obtained 3 hours after rhenium sulfur injections with a conventional gamma camera (Hélix ${ }^{\circledast}$, Elscint, Haïfa), showed one SLN (figure 1). Immediately before surgery, when the patient was in operative position, four images ( 2 minutes data acquisition each) were obtained with the OGC to map the full axillary area (figure 2 represents a picture of the OGC). The precise localization of this SLN and an estimation of its depth were determined from the

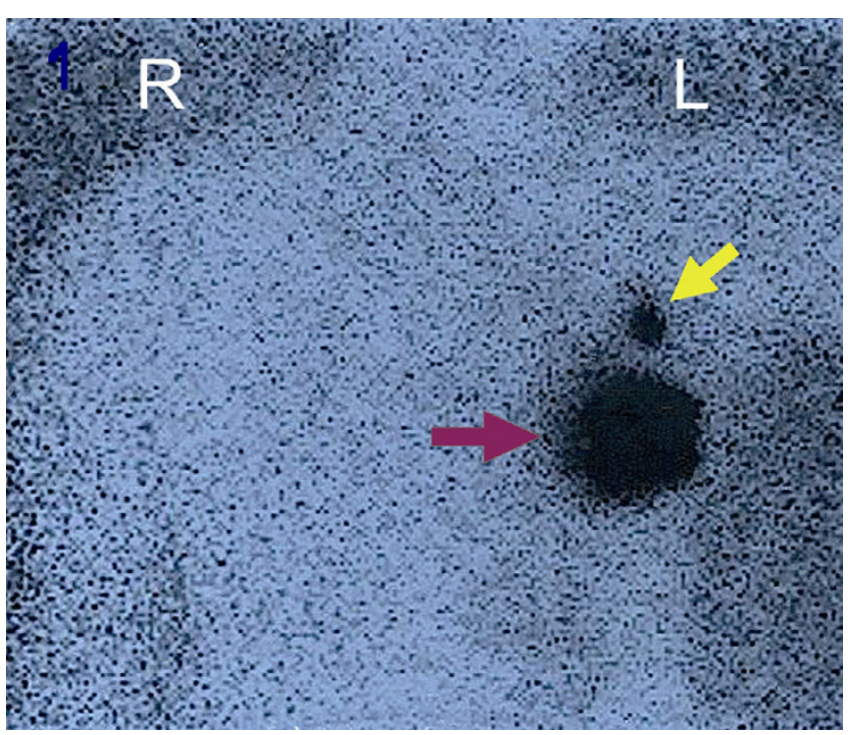

\section{Figure I}

The anterior view of the conventional lymphoscintigraphy shows one SLN indicated by the yellow arrow. The purple arrow indicates the injection site.

data by using the width of the image cumulative profiles (figure 3).

The surgery began with 4 subareolar injections of Methylene Blue (Lab Aguettant ${ }^{\circledR}$, Lyon, France), and the search for the SLN was carried out using the CarolIReS intraoperative probe [13], which detected only one SLN. This SLN was blue stained and its measured activity was 9.6

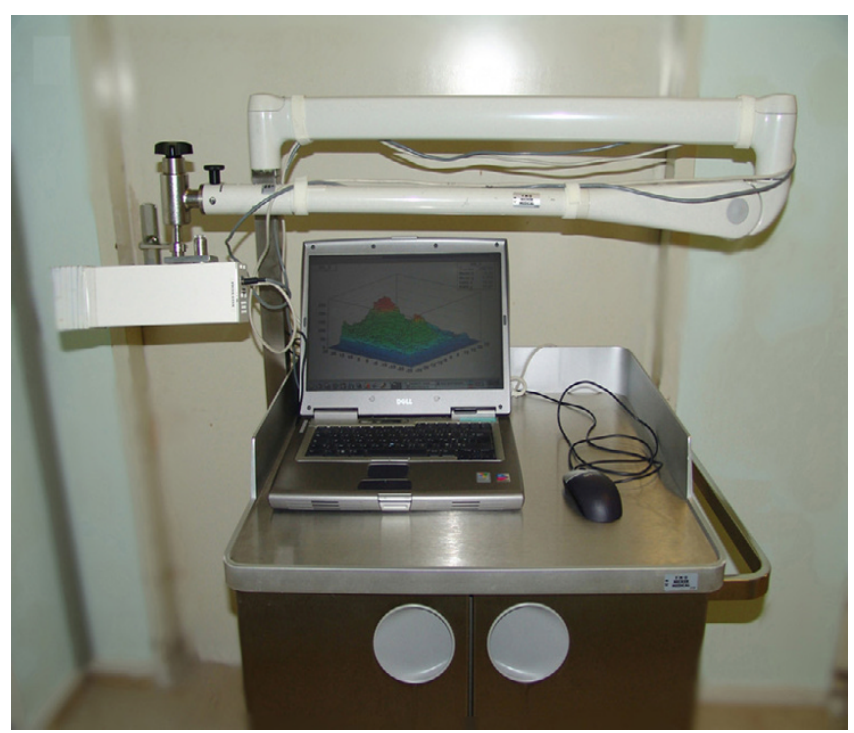

Figure 2

Picture of the CarollReS gamma camera built at the IPHC, Strasbourg, France. 


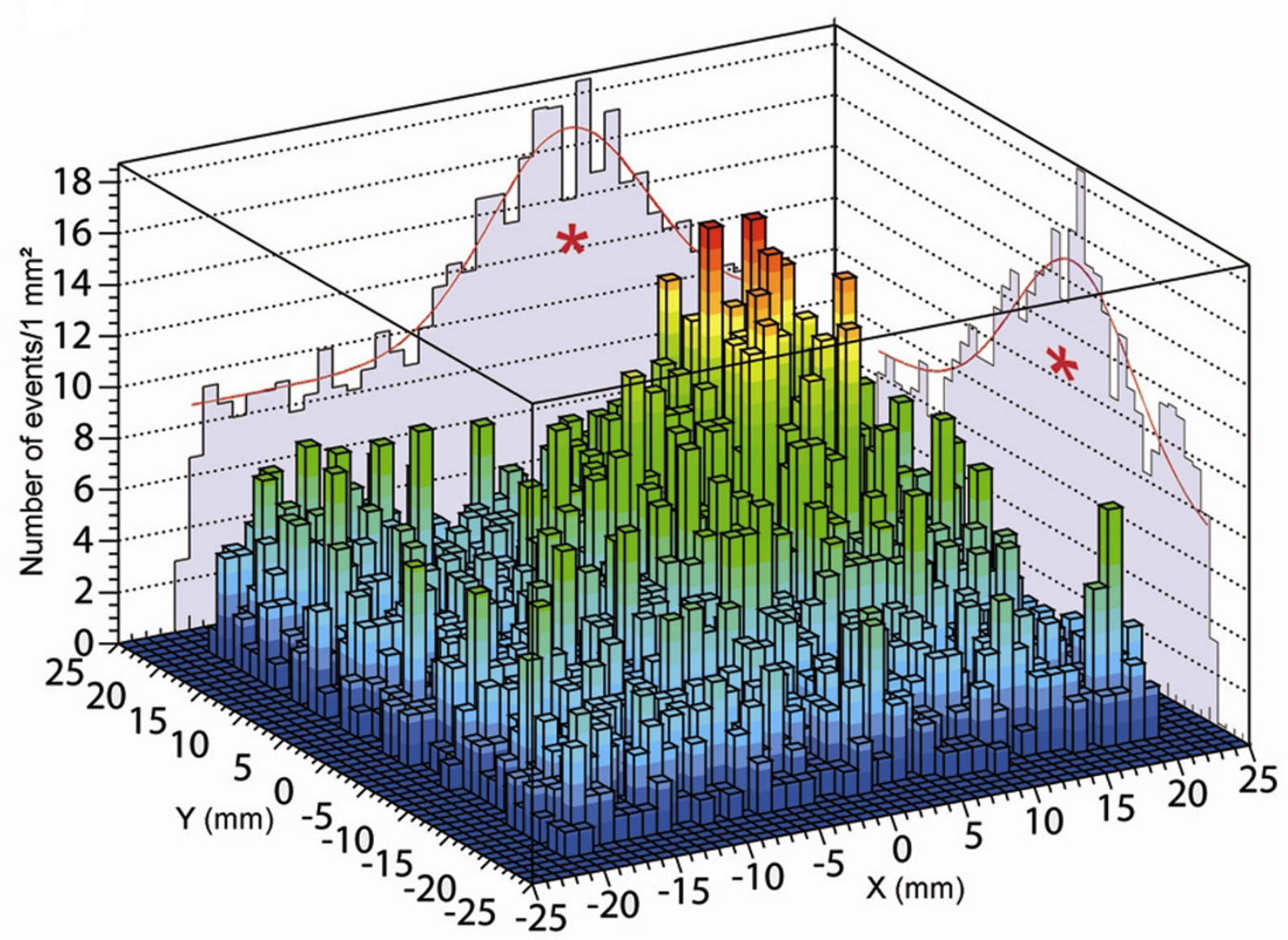

Figure 3

Preoperative image of the axillary area obtained with the CarollReS gamma camera. The $x$ and $y$ cumulative profiles (red stars) are used to determine the planar coordinates and the anatomical depth of the first SLN.

$\mathrm{kBq}(0.26 \mu \mathrm{Ci})$. It was subsequently submitted for frozen section analysis, which showed the absence of cancer cells. During this analysis, the axillary area was thoroughly palpated by the surgeon and no nodes showing signs of extensive tumor involvement were identified. A second exploration of this area was then performed using the OGC enabling the identification of a second SLN located at a distance from the first one of less than the spatial resolution of the OGC (figure 4). This SLN was not stained and had a low activity of $0.5 \mathrm{kBq}(0.01 \mu \mathrm{Ci})$ concentrated in its hilum.

The histological examination revealed an 8-mm macrometastasis occupying the whole structure of this SLN without residual sinusoidal macrophages (figure 5). Axillary clearance was then performed and each of the $11 \mathrm{LN}$ removed was negative. Axillary surgery was followed by lumpectomy. A postoperative OGC image proved that no SLN remained (figure 6) and was considered a quality control document.

\section{Discussion}

This is the first published case in which an OGC enabled the detection of a non-stained metastatic SLN, which was neither diagnosed by conventional lymphoscintigraphy, nor by operative probe, nor by palpation of the axillary area performed by an experienced surgeon. This can be partially explained by the very low activity of this SLN (0.5 $\mathrm{kBq}$ ). It corresponds to $0.02 \%$ of the injected dose, which is inferior to our mean measured value of $0.5 \%$. Metastatic SLN sometimes have very low activity, because they contain numerous cancer cells and few macrophages, the latter being the cells that retain radiocolloïdes [14]. In our experience, the activity of metastatic SLN is less than 10 $\mathrm{kBq}$ in more than half of the cases. So the detection efficiency of OGC must be good, and that of the one we used in this reported case was $2.2 \mathrm{cps} . \mathrm{kBq}^{-1}$. Future clinical trials will have to determine whether or not there is an inferior threshold for the activity of the SLN which should be removed. 


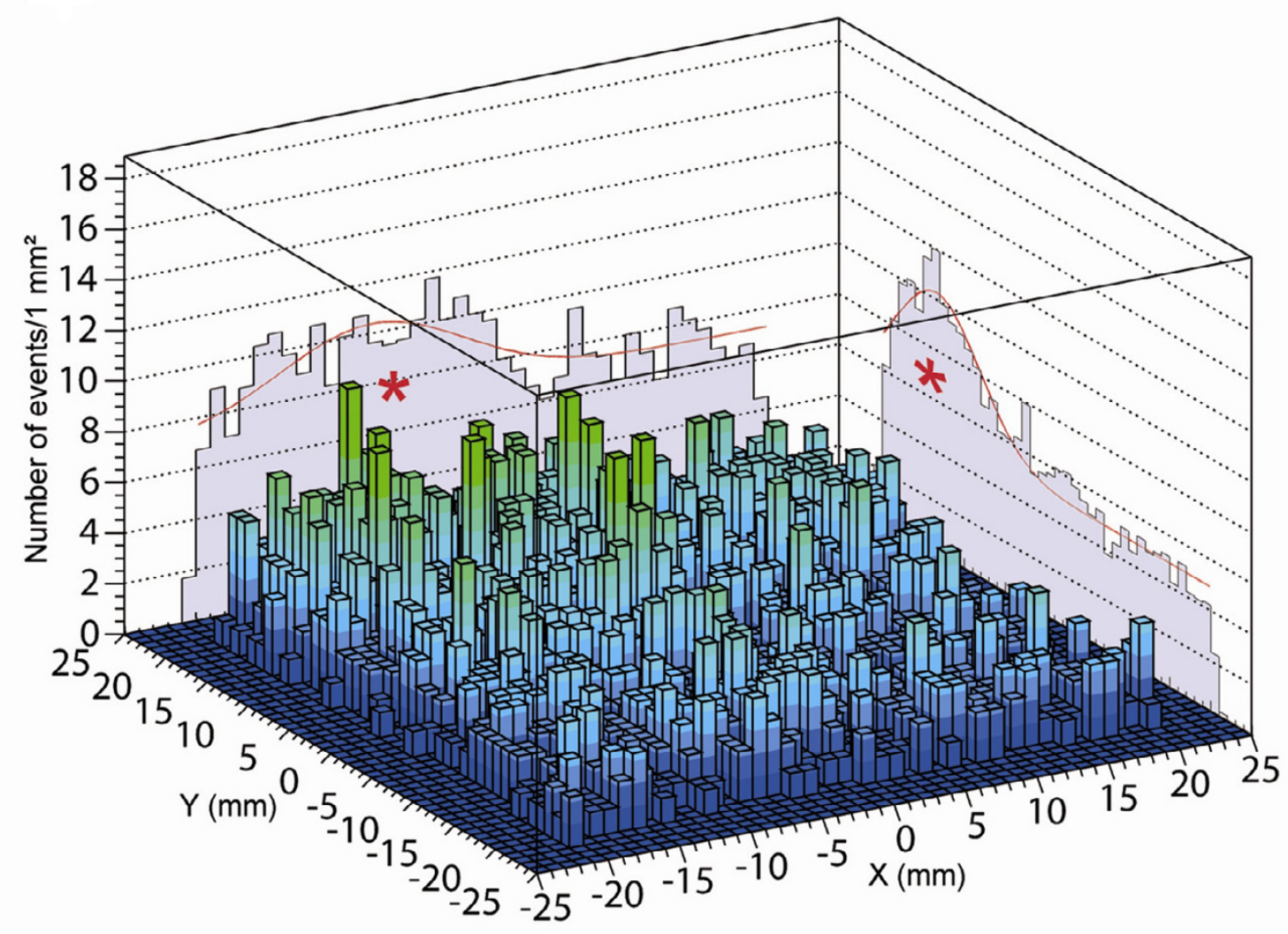

\section{Figure 4}

Peroperative image of the axillary area obtained with the CarollReS gamma camera. The $x$ and $y$ cumulative profiles (red stars) are used to determine the planar coordinates and the anatomical depth of the second SLN.

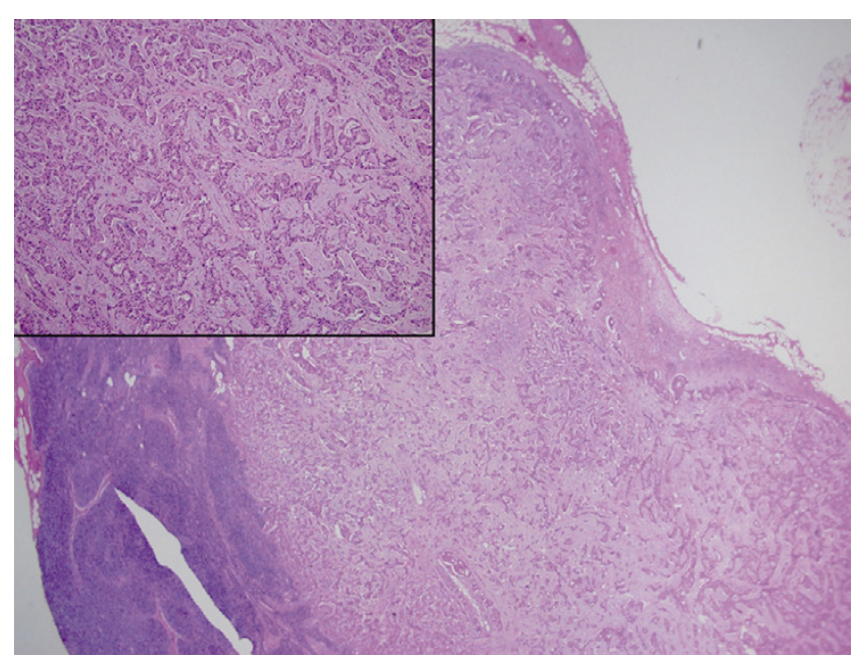

Figure 5

Ductal breast carcinoma metastasis destroying nodal architecture (Hematoxilin and Eosin $\times 20$ ). Inset: metastatic cells (Hematoxilin and Eosin $\times 200$ ) in the second SLN.
In addition, ongoing trials using OGC will have to evaluate if the latter lead to unnecessary SLN removal and increase morbidity or if they decrease the false negative rate (FNR). However, it has been demonstrated that the identification of multiple SLN, when present, reduced the FNR. For example, Goyal et al. [15] studied a series of 803 patients with breast cancer who underwent successful SLN procedure followed by an axillary clearance. In patients who had one SLN harvested, the FNR was $10 \%$, compared with $1 \%$ in patients who had 3 or more SLN removed. Factors associated with finding multiple SLN (62.4\% of patients) were age less than 50 years, low body mass index, tumor in the outer half of the breast, SLN visualization on lymphoscintigraphy and an interval of 12 hours or less between radioisotope injection and SLN procedure. For $99.6 \%$ of node-positive tumors, metastases were detected within the first 4 SLN removed and the authors suggested that the removal of more than 4 SLN was unnecessary [15]. Ongoing clinical trials will have to discuss precisely the usefulness of OGC according to the number of SLN found with conventional procedures (less than or equal to 4 or greater than 4 ). 


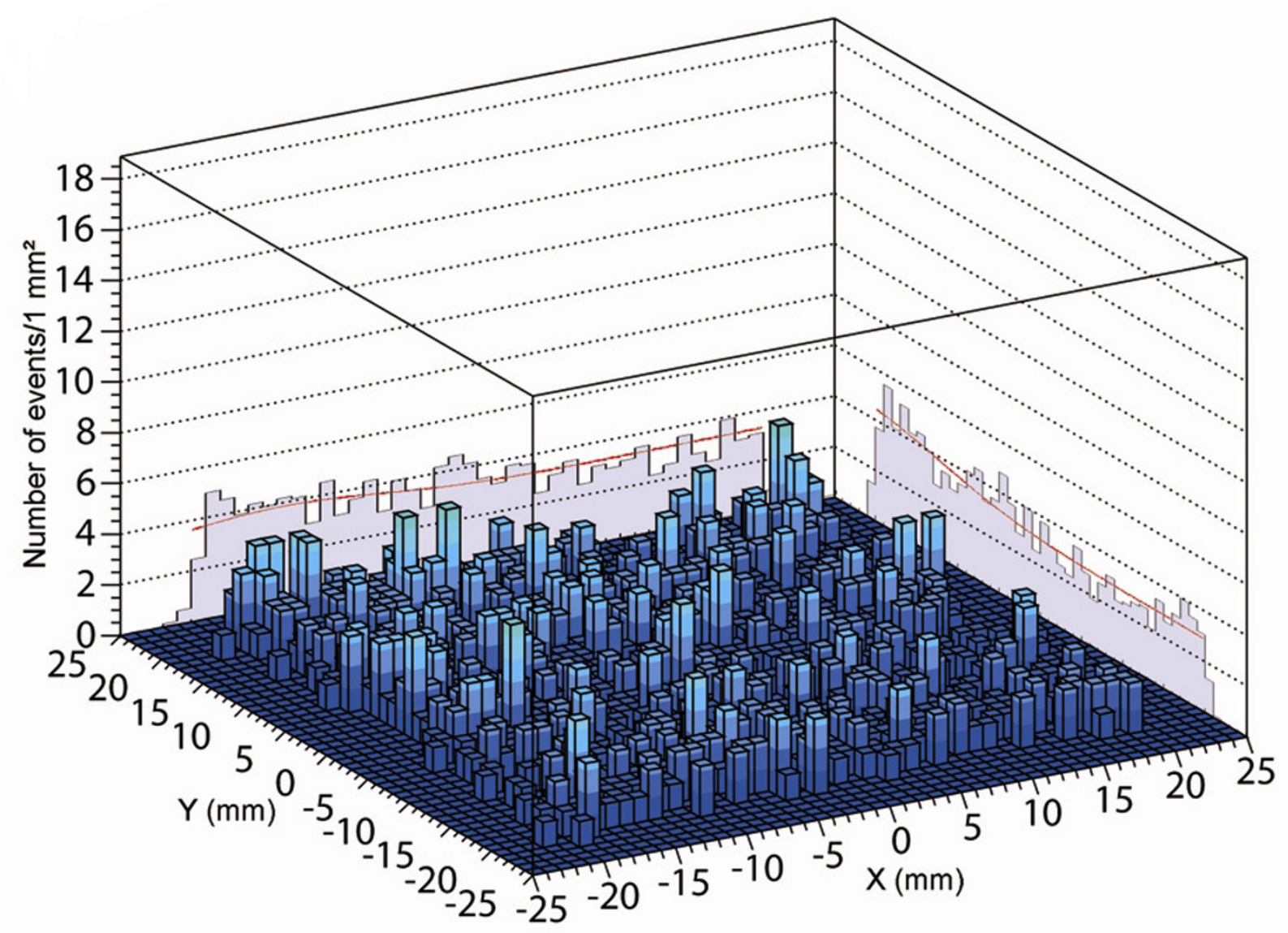

Figure 6

Postoperative image of the axillary area obtained with the CarollReS gamma camera.

\section{Conclusion}

This case report illustrates that an efficient OGC may be able to decrease the risk of FNR of the SLN procedure, and could be an additional tool to control the quality of the surgery. But a precise evaluation of OGC should be based upon data from a large number of patients. Our ongoing clinical trial [11] will be soon published and a new clinical trial, including 100 breast cancer patients, will begin at the end of 2007 to evaluate the usefulness of the new CarolIRes OGC $\left(\mathrm{FOV}: 100 \times 100 \mathrm{~mm}^{2}\right)$ in the SLN procedure.

\section{Abbreviations}

FNR: false negative rate

FOV: field of view
OGC: operative gamma camera

SLN: sentinel lymph node

\section{Competing interests}

The author(s) declare that they have no competing interests.

\section{Authors' contributions}

$\mathrm{CM}$ carried out the clinical trial, performed the surgical treatment and drafted the manuscript. SC performed the pathological analysis and helped to draft the manuscript. NA performed the lymphoscintigraphy and was medical investigator of the clinical trial. JLG, SS and DH were scientific investigators of the clinical trial, helped to draft the manuscript and performed the statistical analysis of the 
clinical trial. All authors read and approved the final manuscript.

\section{Acknowledgements}

We thank Jean-Pierre Bellocq, Jean-Philippe Brettes, Marie-Pierre Chenard, Doris Frankhauser, Philippe Liégeois, Jean-Louis Mehl and Roland Schaeffer for their collaboration and David Brasse and Karl Neuberger for their helpful discussions. This work was supported by funds from the Centre National de la Recherche Scientifique, the Hôpitaux Universitaires de Strasbourg, the University Louis Pasteur and the Région Alsace. We are indebted to the Ligue Nationale Française contre le Cancer and the Comités du Haut-Rhin et du Bas-Rhin for their constant and considerable financial support.

Written consent was obtained from the patient for publication of study.

\section{References}

I. Veronesi U, Paganelli G, Galimberti V, Viale G, Zurrida S, Bedoni M, Costa A, de Cicco C, Geraghty JG, Luini A, Sacchini V, Veronesi P: Sentinel-node biopsy to avoid axillary dissection in breast cancer with clinically negative lymph-nodes. Lancet 1997, 349(9069): I 864-1867.

2. Bleiweiss IJ: Sentinel lymph nodes in breast cancer after $\mathbf{1 0}$ years: rethinking basic principles. Lancet Oncol 2006, 7(8):686-692.

3. Leppanen $E$, Leidenius M, Krogerus L, von Smitten K: The effect of patient and tumour characteristics on visualization of sentinel nodes after a single intratumoural injection of Tc $99 \mathrm{~m}$ labelled human albumin colloid in breast cancer. Eur J Surg Oncol 2002, 28(8):82I-826.

4. Goyal A, Newcombe RG, Chhabra A, Mansel RE: Factors affecting failed localisation and false-negative rates of sentinel node biopsy in breast cancer--results of the ALMANAC validation phase. Breast Cancer Res Treat 2006, 99(2):203-208.

5. Abe A, Takahashi N, Lee J, Oka T, Shizukuishi K, Kikuchi T, Inoue T, Jimbo M, Ryuo H, Bickel C: Performance evaluation of a handheld, semiconductor ( $\mathrm{CdZnTe}$ )-based gamma camera. Eur J Nucl Med Mol Imaging 2003, 30(6):805-8I I.

6. Kopelman D, Blevis I, losilevsky G, Reznik A, Chaikov A, Weiner N, Israel $O$, Hashmonai $M$ : A newly developed intra-operative gamma camera: performance characteristics in a laboratory phantom study. Eur J Nucl Med Mol Imaging 2005, 32(10): $12 \mid 7-1224$.

7. Mathelin C, Salvador S, Huss D, Guyonnet JL: Precise localization of sentinel lymph nodes and estimation of their depth using a prototype intraoperative mini gamma-camera in patients with breast cancer. J Nucl Med 2007, 48(4):623-629.

8. Motomura K, Noguchi A, Hashizume T, Hasegawa Y, Komoike Y, Inaji $H$, Saida T, Koyama H: Usefulness of a solid-state gamma camera for sentinel node identification in patients with breast cancer. J Surg Oncol 2005, 89(I): I 2-17.

9. Pitre S, Menard L, Ricard M, Solal M, Garbay JR, Charon Y: A handheld imaging probe for radio-guided surgery: physical performance and preliminary clinical experience. Eur J Nucl Med Mol Imaging 2003, 30(3):339-343.

10. Tsuchimochi M, Sakahara H, Hayama K, Funaki M, Ohno R, Shirahata T, Orskaug T, Maehlum G, Yoshioka K, Nygard E: A prototype small CdTe gamma camera for radioguided surgery and other imaging applications. Eur J Nucl Med Mol Imaging 2003, 30(I2): 1605-1614.

II. NCT00357487 CTI: Evaluation of Two New Medical Instruments Dedicated to the Sentinel Lymph Node Technique in Case of Breast Cancer. 2006.

12. Salvador S, Bekaert V, Mathelin C, Guyonnet JL, Huss D: An operative gamma camera for sentinel lymph node procedure in case of breast cancer. JINST 2 P07003 2007.

13. Mathelin C, Piqueras I, Guyonnet JL: Performances of a new prototype handheld scintillating probe for the search of sentinel lymph nodes in breast cancer. Eur J Surg Oncol 2006, 32(I):24-28.

14. McCready DR: Intraoperative sentinel node assessment is a touchy subject. Ann Surg Oncol 2002, 9(4):32I-323.
15. Goyal A, Newcombe RG, Mansel RE: Clinical relevance of multiple sentinel nodes in patients with breast cancer. $\mathrm{Br} J$ Surg 2005, 92(4):438-442.
Publish with Biomed Central and every scientist can read your work free of charge

"BioMed Central will be the most significant development for disseminating the results of biomedical research in our lifetime. " Sir Paul Nurse, Cancer Research UK

Your research papers will be:

- available free of charge to the entire biomedical community

- peer reviewed and published immediately upon acceptance

- cited in PubMed and archived on PubMed Central

- yours - you keep the copyright
BioMedcentral 